\title{
Experimental Measurement of Weak Band Intensities
}

\author{
Kevin K. Lehmann and Daniele Romanini \\ Department of Chemistry, Princeton University, Princeton NJ 08544, USA
}

\section{Introduction}

In modeling the opacity of astronomical objects, such as stellar atmospheres, the most important transitions are those with absorbance ${ }^{1}=\alpha L \sim 1$, where $\alpha$ is the absorption coefficient of the atmosphere and $\mathrm{L}$ is the pathlength that light travels in leaving the atmosphere. Transitions with much smaller absorbance are not important, while those with much larger absorbance are effectively opaque. The relevant absorption lengths $L$ are typically orders of magnitude larger than can be realized in the laboratory, and thus obtaining the needed laboratory data is a significant experimental challenge. This volume contains several examples of the power of modern $a b$ initio methods to predict spectra of simple triatomic molecules, such as $\mathrm{H}_{2} \mathrm{O}$, but it is still necessary to at least calibrate the predictions against laboratory bands of similar oscillator strength. The theoretical treatment of molecules with four or more atoms lags far behind the theoretical treatment of triatomics and present experimental capabilities.

The absorption coefficient $\alpha$ can be decomposed into a product

$$
\alpha=N_{0} \sigma^{2}(\lambda)
$$

where $N_{0}$ is the number density (in atoms or molecules per $\mathrm{cm}^{3}$ ) and $\sigma^{2}(\lambda)$ is the absorption cross section. At pressures low enough to ignore pressure broadening, $\sigma^{2}(\lambda)$ is a function of only the species responsible for the absorption and the temperature, and is proportional to the molecular transition dipole moment squared. The absorbance of a transition can be small either because of a small number density or a small cross section. Low number density is typically caused by either an unstable or refractory material, or because the molecule in question contains elements of low abundance. Modern chemical physics offers many methods for dealing with these problems, such as electric discharge (see Lovas et al. 1992, for example) or laser oblation sources (Powers et al. 1982) to produce radicals or small clusters. Sensitive indirect spectroscopic detection methods, such as

1 The absorbance is defined as $\ln T$ where $T$ is the transmission of the atmosphere at a particular wavelength $\lambda$. The absorption coefficient is the absorbance per unit length. The extinction is defined as $\log _{10} T$ and thus is proportional to the absorbance. 
laser induced fluorescence ${ }^{2}$ or resonant enhanced multiphoton ionization ${ }^{3}$ allow spectroscopic detection of as little as $10^{2}$ molecules $/ \mathrm{cm}^{3} /$ quantum state in favorable cases (Fairbanks et al. 1975). While it is hard to combine these methods with high temperatures, because of thermal radiation and ionization, they are important spectroscopic methods that provide a wide range of data important in astrophysics.

Much harder to overcome is limitation imposed by intrinsically weak transitions. The most important of these are high overtone vibrational transitions. The standard harmonic oscillator approximation predicts that light can only change vibrational modes by \pm 1 quanta, and this leads to intense absorption in the IR for all but homonuclear diatomic molecules. Molecules are not, in fact, harmonic oscillators, and real molecules have overtone transitions that change the excitation of vibrational modes by 2,3 , or more quanta, as well as combination bands where two or more modes are simultaneously excited. For hydrogen containing molecules, transitions to levels with between 5 and 8 quanta of the $\mathrm{X}-\mathrm{H}$ stretching excitation fall in the visible and have been known for many years. See the text by Herzberg (1945) for a compilation of the early work. These transitions, however, have cross sections only $10^{-5}-10^{-8}$ as large as the allowed IR fundamentals (Burberry et al. 1979). Such weak transitions are important in the calculation of the opacity of both oxygen-rich and carbon-rich stars, since the long column density of species such as $\mathrm{H}_{2} \mathrm{O}, \mathrm{HCN}$, and $\mathrm{C}_{2} \mathrm{H}_{2}$ give even these weak overtone transitions appreciable absorbance. The many weak lines fill in the spectral windows between the strong lines, and in this way effectively block the stellar light flux.

In this chapter, we will attempt to briefly review the methods that have been used to detect weak overtone transitions. These include absorption measurements in traditional long pathlength cells, photoacoustic spectroscopy, and intracavity loss spectroscopy. We will then describe a new method of high sensitivity and flexibility, ring-down cavity spectroscopy. This method was first introduced by O'Keefe \& Deacon (1988) and has been further developed in our laboratory and used for the study of extremely weak overtone bands of HCN (Romanini \& Lehmann 1993).

\section{Traditional Long-path Spectroscopy}

The traditional approach to increasing the absorbance pathlength is to use a multipass cell to fold the light path many times through the cell. The most important design is that first reported by White (1942), based upon three mirrors, though many modification on this design have been published (Welsh et

\footnotetext{
${ }^{2}$ In laser induced fluorescence, molecular absorption of laser radiation is detected by monitoring emission at right angles to the laser beam.

${ }^{3}$ In resonant enhanced multiphoton ionization, one monitors absorption via detection of ions produced by selective photoionization from an excited electronic state.
} 
al. 1951). A key feature of all these designs is the use of low $F$ number ${ }^{4}$ optics and a placement of the mirrors at a separation that is on the edge between a stable and unstable optical cavity. ${ }^{5}$ This results in a refocusing to a constant spot size on each round trip through the cell, and a running line of spots on one mirror. The pathlength is adjusted by changing the number of spots that fall on this mirror. Large mirrors are needed to keep the light throughput high when such a cell is used with incoherent sources, but also to give ample space to resolve the spots used for coupling the light into and out of the cell. The maximum number of traversals of the cell is limited by both the reflectivity of the mirrors and by the need to physically separate the spots. Practical limits have been on the order of a few hundred passes of the cell, which translates to a maximum pathlength on the order of $0.5 \mathrm{~km}$ for cells of a few meters physical length (Smith et al. 1986). Special, very large cells have been constructed that allow pathlengths of several $\mathrm{km}$ to be reached (Giver 1978), but these are quite expensive and difficult to control in temperature. Because they are not stable optical cavities, density gradients and turbulence caused by convective flow can easily destroy the optical beam quality and lead to substantial noise on the optical transmission. This is particularly a problem when the cells are used with Fourier Transform instruments, but also limits the sensitivity when used with coherent sources.

In recent years, novel cell designs based upon off-axis coupling into a marginally stable, near concentric optical cavity have been used, and are known as Herriott Cells (Herriott et al. 1964). Light enters and leaves the cell through a single hole in one of the two mirrors. Unless the input light is carefully mode matched, the spot size changes on each round trip, but it returns to its original size when it once again passes through the coupling hole. The stable optical cavity makes the cell less sensitive to both mechanical vibration and convection than a White type cell. One minor drawback is that the pathlength is varied by scanning the physical separation of the two mirrors and this can be somewhat difficult in vacuum. The effective $F$ number of a Herriott cell is much greater than a White cell, and thus they are not as efficient for incoherent sources, but for laser sources they are much superior. Like White cells, the maximum pathlength is limited by both the finite reflectivity of the large mirrors required

4 The $\mathrm{F}$ number is the ratio of the focal length to the limiting aperture of an optical system. The light throughput of such a system is proportional to $1 / \mathrm{F}^{2}$.

5 There is not enough room in this chapter to adequately review the theory of optical cavities. See the book by Yariv (1989) for an excellent introduction to this subject. For the uninitiated, it is enough to know that both multipass cells and lasers can be treated as optical cavities which confine light with a certain loss per pass. Cavities are stable or unstable depending on the behaviour of light rays slightly divergent from the optical axis of the cavity. These will remain close to the axis if the cavity in which they propagate is stable, while in an unstable cavity their distance from the axis will diverge exponentially. For a cavity on the edge between stable and unstable, the divergence is linear with the number of passes (think to the case of two plane parallel mirrors). Light is mode matched into a cavity if the light beam has the right size and wavefront to exactly overlap itself on each round trip of the cavity. 
(the beam attenuation through the cell is proportional to the mirrors reflectivity raised to the power of the number of cell traversals) and by the requirement that at each round trip the beam forms a distinct, spatially resolved spot on one of the mirrors. For cells of practical size, these limit the total pathlengths to one the order of $1 \mathrm{~km}$ or less.

\section{Photoacoustic Spectroscopy}

Photoacoustic or optoacoustic spectroscopy monitors absorption by its effect on the sample and thus is an indirect detection method such as laser induced fluorescence. Following absorption of laser light, molecules collisionally relax, releasing heat which increases the local temperature. This local temperature rise leads to expansion, followed by contraction as the heat energy is dissipated. Thus chopping of a laser beam at an acoustical frequency $(\sim 1 \mathrm{kHz})$, will produce a synchronous sound wave which can be detected by a microphone. Photoacoustic cells are built to minimize sound waves produced by window absorption and may include a resonant inner section which allow the formation of acoustic standing waves at the laser chopping frequency. Microphones are extremely sensitive and, with this careful cell design, allow one can detect on the order of $10^{-8} \mathrm{~W} / \mathrm{cm}$ of deposited energy (Kritchman et al. 1978). Since one measures the deposited energy, the signal (and thus sensitivity) increases linearly with average laser power. Thus photoacoustic spectroscopy is typically done with the sample cell inside the optical cavity of a $100 \%$ amplitude modulated, continuous wave laser to maximize the signal strength. A minimum noise equivalent absorption coefficient of $4 \cdot 10^{-10} \mathrm{~cm}^{-1}$ has been obtained in this way (Davidsson et al. 1990). Efficient conversion of the heat to acoustic energy requires pressures of at least $10-100$ torr, but this can be made up principally of buffer gas when the sample vapor pressure is too low.

The principal advantage of photoacoustic spectroscopy is its extreme sensitivity, the highest so far obtained for species that do not fluoresce. A number of drawbacks of photoacoustic spectroscopy are: (1) One must have a quiet acoustic environment; thus use of an electric discharge or rapid flow of the sample leads to a substantial increase in noise. (2) The sample is exposed to high average light flux, which can lead to photochemistry in some situations. (3) One needs a high average power continuous wave laser source, which is higher in cost and has less wavelength flexibility than pulsed laser sources (which are easily frequency doubled in nonlinear crystals and shifted by stimulated Raman scattering into the UV and near IR respectively (Yariv 1989)). (4) The indirect nature of the detection results makes determination of absolute absorption strengths difficult. The only practical way to calibrate the strength of the acoustic signal is to use a mixture of a gas which has some transition whose cross section is already known along with the"gas of interest. Even with such calibration, uncertainties on the order of $20 \%$ have remained (Smith et al. 1987). 


\section{Intracavity loss absorption spectroscopy (ICLAS)}

When a laser with a large gain bandwidth ${ }^{6}$ (such as a dye laser ${ }^{7}$ ) is switched on, light intensity builds up from spontaneous emission. At first, the spectrum matches the gain bandwidth of the laser, but then begins to shift and narrow due to gain narrowing and then mode competition. ${ }^{8}$ If the laser contains a weak intracavity (i.e. inside the optical cavity) absorber, whose absorption features are narrow compared to the laser bandwidth, then as the time during which the laser has been pumped $\left(t_{g}\right)$ increases, narrow "holes" are eaten out of the broad band laser emission spectrum. It has been established that for the first $500 \mu \mathrm{s}$ the strength of these holes have an equivalent absorbance, $A$, of

$$
A=\alpha \cdot f \cdot c \cdot t_{g}
$$

where $\alpha$ is the absorbance coefficient of the gas, $f$ the fraction of the laser cavity filled by the absorber (typically $\sim 0.5$ ) and $c$ the speed of light. Thus one has an effective pathlength, $L_{e f f}$ given by:

$$
L_{e f f}=f \cdot c \cdot t_{g}
$$

which can be up to a maximum of $\sim 100 \mathrm{~km}$. Beyond this generation time $t_{g}$, mechanical vibrations and other sources of dephasing cause the depth of the absorption features to no longer follow a simple Lambert-Beer's law (Stoeckel \& Atkinson 1985). Timing is achieved by the use of acousto-optic modulators on the pump and output of the laser, and the laser output is dispersed on a large spectrograph with an array detector. Using this technique, it is possible to measure spectroscopic features with an absorption coefficient as low as a few times $10^{-8} \mathrm{~cm}^{-1}$ (Stoeckel et al. 1982).

The sensitivity of this method is not quite as high as photoacoustic spectroscopy, but is much higher than that obtained with traditional long path cells. As long as one is careful to remain in the early time region, the absorption coefficient, and thus the cross section (assuming one knows the gas density and cavity fill factor), can be extracted from the optical depth of the observed absorption features. Because of the pulsed nature of the experiment, and the near threshold operation of the laser, chances for inadvertent photochemistry are reduced compared to intracavity photoacoustic spectroscopy. The chief disadvantages are that this method requires complex instrumentation. One must use a custom designed continuous wave dye laser that has been carefully designed to remove all sources of stray interference which can lead to narrow bandwidth spikes on the laser output spectrum. In order to achieve Doppler limited resolution, one must use a spectrograph of extremely high $\left(\sim 10^{6}\right)$ dispersion, which is an expensive piece of custom instrumentation.

6 The gain bandwidth is the spectral region over which a laser has net gain on each round trip.

7 A dye laser uses a solution of an organic dye that is optically pumped by another laser to produce gain. The principal advantage of these lasers is that they can be tuned over a broad spectral region.

${ }^{8}$ These phenomena are discussed in the text by Yariv (1989). 


\section{$5 \quad$ Ring-Down Cavity spectroscopy}

Ring down cavity spectroscopy is based upon a simple idea which has become practical due to recent advances in reducing loss in dielectric mirrors. A ring down cavity is made from two highly reflective, concave mirrors aligned as a stable, low loss optical cavity (i.e. the separation less than twice the radius of curvature). In our own work, radii of $1-2 \mathrm{~m}$ and separation $\mathrm{L}=1.5-3 \mathrm{~m}$ are typical. It is now possible to purchase at low cost $(\sim \$ 100$ each) small mirrors with reflectivity $\mathrm{R}>99.99 \%$ over a range of some $60 \mathrm{~nm}$ anywhere in the visible, and mirrors with $\mathrm{R}=99.9998 \%$ have been reported near $840 \mathrm{~nm}$ (Rempe et al. 1992). Light from a conventional pulsed dye laser is coupled into the ring down cavity through one end. If the length of the laser pulse is less than the round trip time of the cavity $(2 L / c=10-20 \mathrm{nsec})$, then there can be no interference and a small but stable fraction of the incident light $\left(\sim 10^{-5}\right)$ enters the cavity. For a typical input pulse energy of a modest $1 \mathrm{~mJ}$, this corresponds to $\sim 3 \cdot 10^{10}$ photons. These photons are trapped between the high reflectivity mirrors and slowly decay due to the combined loss of the mirrors and any molecular absorber located between the mirrors. The empty cell has a decay time $\tau=L / c(1-R)$. On each round trip, something like $10^{-5}$ of the intracavity light intensity is transmitted through the back mirror and is detected by a photomultiplier tube (PMT). With the time constant of the PMT set long compared to the round trip time, its output current follows a smooth exponential decay. O'Keefe \& Deacon (1988) digitized this complete curve and did a least squares fit to extract the decay rate. In our own work (Romanini \& Lehmann 1993), we have found that a much simpler system of a double gated integrator, which averages the light intensity in two time windows separated by $\Delta t \sim 2 \tau$, works essentially as well. The gated integrator output, $S$, is proportional to the ln of the ratio of the signals in each of the two windows, which is given by:

$$
S=\ln \left[\frac{A}{B}\right]=-\frac{\Delta t}{\tau}-L_{\mathrm{eff}} \boldsymbol{\alpha}
$$

where $\tau$ is the empty cavity decay time given above, $L_{\mathrm{eff}}=c \Delta t$ is the effective cavity length (how far the light traveled between the two times that the gated integrator samples the light intensity leaving the ring down cavity) and $\alpha$ is the absorption coefficient of the gas in the cell. In our work with $\lambda=570-435 \mathrm{~nm}$, we have achieved effective pathlengths as large as $60 \mathrm{~km}$, near the maximum attained by the ICLAS method. Figure 1 shows a schematic of the apparatus used. Since the signal is the In of the ratio of the light intensity at early and late times, shot-to-shot fluctuations in the laser intensity are normalized out. In practice we have achieved noise levels of about $0.005\left(2 \sigma_{S}\right)$, which corresponds to a noise equivalent absorption coefficient of $\sim 8 \cdot 10^{-10} \mathrm{~cm}^{-1}$. This is already quite competitive with the best that has ever been achieved by photoacoustic spectroscopy, and significantly better than the claimed sensitivity of ICLAS. We have estimated that with mirrors of 1 ppm loss, such as has been reported by Rempe et al. (1992), a pathlength of $4000 \mathrm{~km}$ and noise equivalent absorption coefficient on the order of $10^{-12} \mathrm{~cm}^{-1}$ should be attainable, which is orders of 


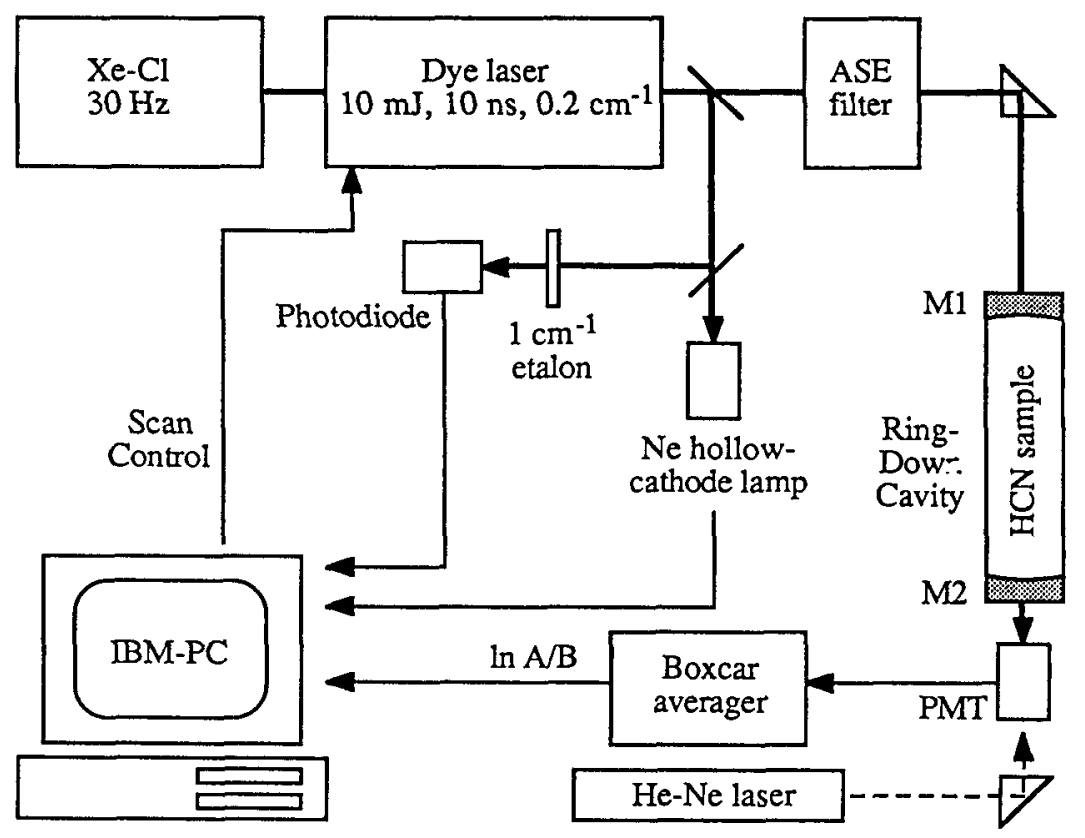

Fig. 1. Schematic of the experimental apparatus. The ASE filter is a stimulated Brillouin scattering cell for filtering the dye laser output of residual amplified spontaneous emission. The HeNe laser is used for rapid alignment of the Ring-Down cavity. Figure taken from Romanini \& Lehmann (1993).

magnitude better than has ever been realized in any absorption based detection method. At one atmosphere pressure, this corresponds to an absorption cross section of $3 \cdot 10^{-32} \mathrm{~cm}^{2}$ ! Since the method is based upon light traveling a known distance through a passive optical cavity, Lambert-Beer's law should hold quantitatively for all pathlengths, and thus the method provides a direct determination of the optical extinction coefficient at a known pathlength.

Compared with the other methods which we have discussed above, the ring down cavity spectroscopy is the simplest and least expensive to implement. It is also the most flexible. It is important to point out that the optics used are of such high reflectivity and modest cost because they are of small size; the coated surfaces are less than $1 \mathrm{~cm}$ in diameter. It is also possible to combine this method with pulsed molecular beam sources (O'Keefe et al. 1990), or with transient species produced in a discharge. The method samples only a narrow pencil of the sample, with a cross section less than $1 \mathrm{~mm}^{2}$. By putting a furnace around the ring down cavity, it should be possible to study high temperature species, something that likely will prove useful for stellar astronomy. Because of the use of a stable optical cavity, small deviations of the light beam due to density gradients will average out over several round trip, and should not contribute to noise as in traditional long pathlength cells. Furthermore, since 
the cross sectional area of a ring down cell is much less than for a White cell, gas turbulence due to convection is much less likely to occur.

\section{HCN Overtone Spectrum}

Using the method of photoacoustic spectroscopy, we had previously investigated the spectrum of HCN for $\lambda$ from $700-540 \mathrm{~nm}$ (Smith et al. 1987), which included bands with up to six quanta of $\mathrm{CH}$ stretch, as well as combination bands of the $\mathrm{CH}$ and $\mathrm{CN}$ stretching modes. The wavelength region was limited by the tuning range of a dye laser pumped by a visible $\mathrm{Ar}^{+}$ion laser. Attempts to determine absolute absorption strengths using the photoacoustic method proved disappointing. We also studied the near-IR spectrum from $1850-660 \mathrm{~nm}$ by using a 6 meter base length White cell (allowing absorption pathlengths up to $432 \mathrm{~m}$ ) with the 1-m Fourier transform spectrometer at the McMath National Solar Observatory at Kitt Peak (Smith et al. 1989).

In order to push the HCN measurements to higher energy, and weaker bands, we have measured the overtone spectrum of HCN for $\lambda=570-430 \mathrm{~nm}$ using a ring down cavity (Romanini \& Lehmann 1993). Figure 2 shows the rotational

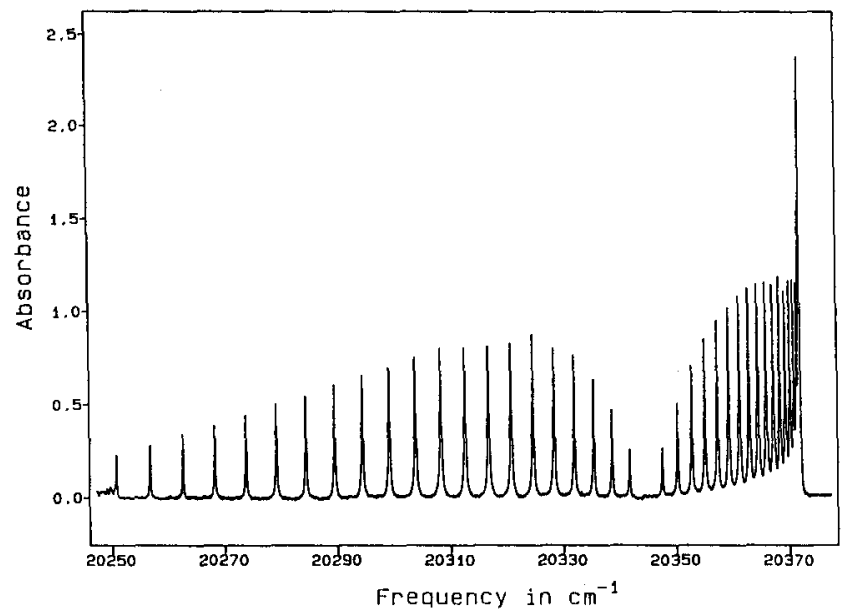

Fig. 2. The spectrum of the $(1,0,6) \leftarrow(0,0,0)$ overtone band of HCN (100 torr, $\Delta t=80 \mu \mathrm{s}, L=24 \mathrm{~km}$ ). Near the last displayed line of the P-branch is the beginning of the $(1,0,6) \leftarrow(0,1,0)$ hot band. Figure taken from Romanini \& Lehmann (1993).

structure of one of the observed bands, the $(1,0,6) \leftarrow(0,0,0)$ band at $491.5 \mathrm{~nm}$, obtained with an effective pathlength of $24 \mathrm{~km}$. In this wavelength region, we have observed and analyzed nine bands of the principal isotopic species (for an analysis on this spectrum see Romanini \& Lehmann 1993). Table 1 contains a 
list of the observed band origin and integrated band intensity ${ }^{9}$ for each transition. Also, for comparison, are listed two predictions for the transition intensities. Both are calculated from two dimensional variational wavefunctions using the $a b$ initio dipole function of Botschwina (1988). The column labeled Botschwina contain the intensities calculated by Botschwina (1992) using his corrected ab initio potential (Botschwina 1988). These calculations used the CEPA-1 method (coupled electron pair approximation). The second column, labeled Carter-HandyMills, contains intensities calculated by us, using the recently determined empirical HCN potential reported by these authors (Carter et al. 1993). Overall, the agreement between theory and experiment is excellent, especially considering the weakness of these bands. For comparison, the $\mathrm{CH}$ stretching fundamental of HCN has a strength of $6 \times 10^{6} \mathrm{~cm} / \mathrm{mol}$ (Smith et al. 1981), nearly $10^{8}$ times larger than the weakest of the bands we have presently observed (Romanini \& Lehmann 1993). These measurements provide a critical test of the accuracy of theoretical calculations, which can easily be used to simulate the spectrum of $\mathrm{HCN}$ even at temperatures of stellar atmospheres. As this book was going to press, Botschwina (1993) reported new results with a dipole function calculated at a higher level of theory, the CCSD(T) method (coupled clusters with single, double and an approximate treatment of triple exciations). The vibrational wavefunctions are unchanged from those he had calculated with his earlier (CEPA-1) potential. The intensities he calculated with this new dipole function are reported in the last column of the table, under the heading CCSD(T). It is seen that the agreement with experiment is substantially improved, with the remaining dif-

Table 1. Comparison of observed and predicted band intensities of the overtone bands of $\mathrm{HCN}$. Observed band origins are accurate to $\pm 0.03 \mathrm{~cm}^{-1}$ intensities to $\pm 5 \%$. The three theoretical predictions are described in the text.

\begin{tabular}{l|lllll}
\hline \hline Transition & $\begin{array}{l}\text { Frequency } \\
\mathrm{cm}^{-1}\end{array}$ & $\begin{array}{l}\text { Exp. inten- } \\
\text { sity cm/mole }\end{array}$ & $\begin{array}{l}\text { Botschwina } \\
\text { CEPA }\end{array}$ & $\begin{array}{l}\text { Carter- } \\
\text { Handy-Mills }\end{array}$ & CCSD(T) \\
\hline $105 \leftarrow 000$ & 17550.42 & 3.51 & 4.53 & 5.4 & 3.62 \\
$006 \leftarrow 000$ & 18377.03 & 2.61 & 2.88 & 3.4 & 2.61 \\
$205 \leftarrow 000$ & 19528.57 & 0.58 & 0.39 & 0.56 & 0.52 \\
$116 \leftarrow 010$ & 20222.27 & 0.78 & & & \\
$106 \leftarrow 000$ & 20344.51 & 0.89 & 0.69 & 0.94 & 0.85 \\
$007 \leftarrow 000$ & 21116.31 & 0.55 & 0.28 & 0.40 & 0.37 \\
$305 \leftarrow 000$ & 21486.77 & 0.092 & 0.026 & 0.052 & 0.073 \\
$206-000$ & 22292.02 & 0.22 & 0.092 & 0.16 & 0.20 \\
$107 \leftarrow 000$ & 23047.11 & 0.24 & 0.098 & 0.17 & 0.20 \\
\hline \hline
\end{tabular}

9 The integrated band intensity $S$, given in units of $\mathrm{cm} / \mathrm{mole}$, is related to the cross section by $S=N_{A} \int \sigma^{2}(\nu) d \nu$ where $N_{A}$ is the Avogadro constant, $\nu$ is the frequency in wavenumber units $\left(\mathrm{cm}^{-1}\right)$, and the integral extends over all the rotational lines in a given vibrational band. The more common unit of integrated band intensity is $\mathrm{km} / \mathrm{mole}=10^{5} \mathrm{~cm} /$ mole and is used because typical allowed IR fundamentals have strength of a few tens to hundreds of $\mathrm{km} /$ mole. 
ferences being in many cases within experimental uncertainties. One can only assume that with vibrational wavefunctions calculated using the higher level of theory that the accuracy will improve further.

\section{Conclusions}

We have here reviewed the methods for obtaining spectra of molecules in regions of very small cross sections, and compared the established methods with the recently developed method of ring-down cavity spectroscopy. The latter method has many advantages over the others, and will likely prove to be widely useful in obtaining spectra of importance for astrophysics.

\section{References}

Botschwin a P., 1988, J. Chem. Soc. Faraday Trans. 2, 84, 1263

Botschwin a P., 1992, private communication

Botschwin a P., Seeger S., Horn M., Fluegge J., Oswald M., Mladenowic M., Hoeper 'U., Oswald R., Schick E., 1993, Proceedings of the 50'th international meeting of the Division de Chimie Physique de la Societe Francaise de Chimie (Mont SainteOdilie), to be published with the American Institute of Physics.

Burberry M.S., Morrell J.A., Albrecht A.C., Swofford R.L., 1979, J. Chem. Phys., 70, 5522

Carter S., Handy N.C., Mills I.M., 1993, J. Chem. Phys., 99, 4379

Davidsson J., Gutow J.H., Zare R.N., 1990, J. Phy. Chem., 94, 4069

Fairbanks W.M., Hänsch T.W., Schawlow A.L., 1975, J. Opt. Soc. Am., 65, 199

Giver L.P., 1978, J. Quant. Spectrosc. Radiat. Transfer, 19, 311

Herriott D.R., Kogelnik H., Kompfner R., 1964, Appl. Opt., 3, 523

Herzberg G., 1945, Infrared and Raman Spectra of Polyatomic Molecule, Van Nostrand

Kritchman E., Shtrikman S., Slatkine M., 1978, J. Opt. Soc. Am., 68, 1257

Lovas F.J., Suenram, Ogata T., Yamanoto S., 1992, A strophys. J., 399, 325

O'Keefe A., Deacon D.A.G., 1988, Rev. Sci. Instrum., 59, 2544

O'Keefe A., Scherer J.J., Cooksy A.L., Sheeks R., Heath J, Saykally R.J, 1990, Chem. Phys. Lett., 172, 214

Powers,D.E., Hansen S.G., Geusic M.E., Puiu A.C., Hopkins J.B., Dietz T.G., Duncan M.A., Langridge-Smith P.R.R., Smalley R.E., 1982, J. Phys. Chem., 78, 1627

Rempe G., Thompson R.J., Kimble H.J., Lalezari R., 1992, Optics. Letters, 17, 363

Romanini D., Lehmann K.K., 1993, J. Chem. Phys., 99, 6287

Smith A.M., Lehmann K.K., Klemperer K., 1986, J. Chem. Phys., 85, 4958

Smith A.M., Jørgensen U.G., Lehmann K.K., 1987, J. Chem. Phys., 87, 3034

Smith A.M., Coy S.L., Klemperer K., Lehmann K.K., 1989, J. Mol. Spectrosc., 134, 134

Smith I.W.M., 1981, J. Chem. Soc. Faraday Trans. 2, 77, 2357

Stoeckel F., Atkinson G.H., 1985, Appl. Optics., 24, 3591

Stoeckel F., Melieres M.-A., Chenevier M., 1982, J. Chem. Phys., 76, 2191

Welsh H.L., Cummining C., Stansbury E.J., 1951, J. Opt. Soc. Am., 41, 712

White J.U., 1942, J. Opt. Soc. Amer., 32, 285

Yariv A., 1989, Quantum Electronic, Wiley and Sons 